\title{
Plantas nectaríferas utilizadas por duas espécies de Melipona da Amazônia
}

$$
\begin{gathered}
\text { BIBLIOTECA } \\
\text { do } \\
\text { INPA }
\end{gathered}
$$

\author{
Maria Lúcia Absy ( $\left.{ }^{*}\right)$ \\ Eronildo Braga Bezerra (") \\ Warwick E. Kerr ${ }^{(*)}$ )
}

\section{Resumo}

Analisa-se o néctar de 302 abelhas de Melipona seminigra merrillae Cock. e 302 de abelhas de Melipona rufiventris paraensis Ducke, durante o período de maio de 1977 a abril de 1978, em Manaus. Do total de amostras estudadas de $\mathbf{M}$. seminigra e $\mathbf{M}$. rufiventris, $16(5,3 \%)$ e $11(3,6 \%)$ respectivamente, não continham pólen. Das 286 restantes $(94,7 \%)$ de M. seminigra, 80 $(28 \%)$ apresentavam pólen de uma só espécie, 88 $(30,8 \%)$ de duas espécies e $118(41,2 \%)$ de três ou mais. De $291(96,4 \%)$ restantes de $\mathbf{M}$. rufiventris, $33(11,3 \%)$ apresentavam pólen de uma só espécie, $54(18,6 \%)$ de duas espécies e $204(70,1 \%)$ de três ou mais. Os grãos de pólen encontrados nas amostras de néctar representam ao todo 60 tipos morfológicos, distribuídos em 29 familias, pelo menos 42 gêneros diferentes, dos quais 39 foram separados ao nível de espécie. Sugere-se que a maior porcentagem de visitas, a mais de uma espécie de plantas nectaríferas $(72 \%)$ do que a plantas poliniferas $(39 \%)$, seja devida a estratégias das plantas unissexuais para obrigar a abelha visitar plantas masculinas para pólen na manhã e femininas para néctar mais tarde.

\section{INTRODUÇÃO}

Foi publicado, em 1977 (Absy \& Kerr, 1977), um estudo sobre 33 espécies de plantas visitadas para obtenção de pólen, durante o período de um ano (agosto/1975 a julho/1976), por operárias de Melipona seminigra merrillae Cock., no Campus do INPA (Manaus).

Como subproduto da investigação, verificou-se que $61 \%$ das operárias que coletavam pólen visitavam apenas uma espécie de flor, $23,3 \%$ visitavam duas e $15,9 \%$ visitavam três ou mais.

O presente trabalho é uma continuação daquele, tendo por alvo determinar as plantas nectaríferas, que são as que contribuem para a produção de mel: produção de mel das duas espécies mais promissoras para a meliponicultura na Amazônia (Kerr et al., 1967).

\section{MAterial e MÉtodos}

Usaram-se duas espécies de abelhas: a mesma Melipona seminigra merrillae Cock., da publicação anterior, e a Melipona rufiventris paraensis Ducke.

Diariamente, às 9 horas, uma abelha de $M$. seminigra e uma de $M$. rufiventris, que retornavam da mata aparentando ter o abdômen dilatado, eram aprisionadas e, por leve pressão no abdômen, fazia-se com que a abelha revertesse o néctar num tubo de centrifugação, ao qual se acrescentavam, depois, $2 \mathrm{ml}$ de ácido acético glacial.

O material era, em seguida, centrifugado duas vezes no ácido acético glacial e preparado para análise pelo método da acetólise (Erdtman, 1960). Após a preparação, os grãos de pólen eram montados em gelatina glicerinada.

Quanto à identificação dos grãos de pólen, era a mesma feita baseando-se nas lâminas de pólen da flora local, na literatura (Absy \& Kerr, 1977; Absy, 1979; Carreira, 1976) e por comparação com a coleção de pólen do INPA.

\section{Resultados}

Os resultados serão apresentados de três maneiras: a) análise dos dados mensais nas tabelas I e II; b) as plantas visitadas por Melipona seminigra e c) as plantas visitadas por Melipona rufiventris.

(*) - Instituto Nacional de Pesquisas da Amazônia, Manaus

(**) - Endereço atual: Departamento de Genética, Faculdade de Medicina de Ribeiräo Preto, 14.100. SP 
TABELA 1 - Dados mensais para Melipona seminigra

\begin{tabular}{|c|c|c|c|c|c|c|c|c|c|c|c|c|}
\hline PLANTAS VISITADAS & $05 / 77$ & $06 / 77$ & $07 / 77$ & $08 / 77$ & $09 / 77$ & $10 / 77$ & $11 / 77$ & $12 / 77$ & $01 / 78$ & $02 / 78$ & $03 / 78$ & $04 / 78$ \\
\hline $\begin{array}{l}\text { AMARANTHACEAE } \\
\text { Alternanthera sp. } \\
\text { ANACARDIACEAE }\end{array}$ & - & + & + & 一 & + & + & + & - & 一 & + & - & - \\
\hline "Anacardium occidentale & - & - & - & - & + & - & - & - & - & - & - & - \\
\hline $\begin{array}{l}\text { Tapirira guianensis } \\
\text { APOCYNACEAE }\end{array}$ & + & - & 一 & + & + & + & - & - & - & - & - & - \\
\hline $\begin{array}{l}\text { Rauwolfia grandiflora } \\
\text { ARALIACEAE }\end{array}$ & - & - & - & - & - & 一 & + & - & - & 一 & 一 & - \\
\hline $\begin{array}{l}\text { - Didymopanax morototoni } \\
\text { BIXACEAE }\end{array}$ & - & + & - & 一 & 一 & - & 一 & - & - & - & 一 & 一 \\
\hline $\begin{array}{l}\text { * Bixa orellana } \\
\text { BORAGINACEAE }\end{array}$ & - & - & - & 一 & - & + & + & - & + & + & - & + \\
\hline $\begin{array}{l}\text { Cordia sellowiana } \\
\text { BURSERACEAE }\end{array}$ & - & + & + & 一 & 一 & - & - & - & - & - & - & 一 \\
\hline $\begin{array}{l}\text { Protium heptaphyllum } \\
\text { COMPOSITAE }\end{array}$ & + & + & + & + & + & + & + & + & 一 & + & + & + \\
\hline Ageratum conyzoides & - & + & + & - & 一 & - & - & - & - & 一 & 一 & - \\
\hline Melampodium camphoratum & - & - & - & 一 & - & - & - & 一 & - & - & - & + \\
\hline - Mikania roraimensis & - & 一 & + & - & - & - & - & - & - & - & - & + \\
\hline $\begin{array}{l}\text { Vernonia scabra } \\
\text { EUPHORBIACEAE }\end{array}$ & - & - & + & + & + & + & - & 一 & 一 & - & - & 一 \\
\hline "Alchornea discolor & + & + & 一 & - & + & + & + & + & + & 一 & - & - \\
\hline $\begin{array}{l}\text { Croton lanjouwensis } \\
\text { Hevea brasiliensis }\end{array}$ & - & - & - & - & - & - & - & $\begin{array}{l}+ \\
+\end{array}$ & + & - & - & - \\
\hline $\begin{array}{l}\text { Mabea nitida } \\
\text { FLACOURTIACEAE }\end{array}$ & - & - & - & - & 一 & + & - & 一 & - & - & $\rightarrow$ & - \\
\hline $\begin{array}{l}\text { Casearia arborea } \\
\text { GUTTIFERAE }\end{array}$ & - & 一 & - & - & 一 & 一 & + & + & 一 & - & - & 一 \\
\hline Clusia aff. nemorosa & - & 一 & - & 一 & 一 & - & - & + & + & 一 & 一 & - \\
\hline $\begin{array}{l}\text { Vismia guianensis } \\
\text { LABIATAE }\end{array}$ & + & 一 & - & + & + & + & + & 一 & - & - & 一 & + \\
\hline $\begin{array}{l}\text { Hyptis brevipes } \\
\text { LEG. CAESALP. }\end{array}$ & + & + & 一 & + & + & 一 & - & - & 一 & - & - & + \\
\hline - Cassia reticulata & + & + & 一 & - & - & - & 一 & + & 一 & 一 & - & 一 \\
\hline $\begin{array}{l}\text { Cassia siamea } \\
\text { LEG. MIMOS. }\end{array}$ & + & - & - & - & + & - & - & - & + & + & + & + \\
\hline "Inga edulis & + & + & + & + & - & 一 & - & 一 & 一 & 一 & 一 & + \\
\hline Mimosa invisa & + & + & - & 一 & 一 & 一 & 一 & - & 一 & - & + & + \\
\hline Mimosa pudica & + & + & - & - & - & - & - & - & + & + & + & $\stackrel{+}{-}$ \\
\hline $\begin{array}{l}\text { Stryphnodendron guianensis } \\
\text { LEG. PAP. }\end{array}$ & + & + & - & - & + & - & - & - & - & - & & \\
\hline $\begin{array}{l}\text { Aeschynomene sensitiva } \\
\text { LORANTHACEAE }\end{array}$ & - & - & - & 一 & + & + & - & - & + & - & - & 一 \\
\hline $\begin{array}{l}\text { Phthirusa micrantha } \\
\text { MALPIGHIACEAE }\end{array}$ & - & 一 & - & 一 & - & - & - & - & - & - & - & + \\
\hline $\begin{array}{l}\text { Byrsonima sp } \\
\text { MELASTOMATACEAE }\end{array}$ & + & 一 & - & - & - & + & + & - & + & - & + & 一 \\
\hline " Bellucia grossularioides & + & + & + & + & + & - & - & - & - & - & 一 & - \\
\hline " Bellucia imperialis & + & + & + & 一 & - & - & - & 一 & - & - & - & 一 \\
\hline $\begin{array}{l}\text { Miconia sp. } \\
\text { MORACEAE }\end{array}$ & + & + & + & + & + & + & + & + & + & + & + & + \\
\hline $\begin{array}{l}\text { Cecropia sp. } \\
\text { MYRTACEAE }\end{array}$ & + & + & + & 一 & 一 & + & + & + & + & + & + & + \\
\hline $\begin{array}{l}\text { "Eugenia sp. } \\
\text { Syzygium jambolana }\end{array}$ & + & $\begin{array}{l}+ \\
+\end{array}$ & - & - & $\stackrel{+}{-}$ & + & + & + & + & + & + & + \\
\hline
\end{tabular}




\section{PALMAE}

- Maximiliana martiana

RUBIACEAE

Borreria capitata

- Borreria laevis

- Borreria latifolia

- Warszewiczia coccinea

SAPINDACEAE

- Talisia sp.

SOLANACEAE

Solanum $s p$.

ULMACEAE

* Trema micrantha

VERBENACEAE

Stachytarpheta cayennensis

- LEGUMINOSAE

PROTEACEAE

Indeterminados:

- Diporado escabrado

Tricolporado reticulado tipo 2

Tricolporado reticulado tipo 3

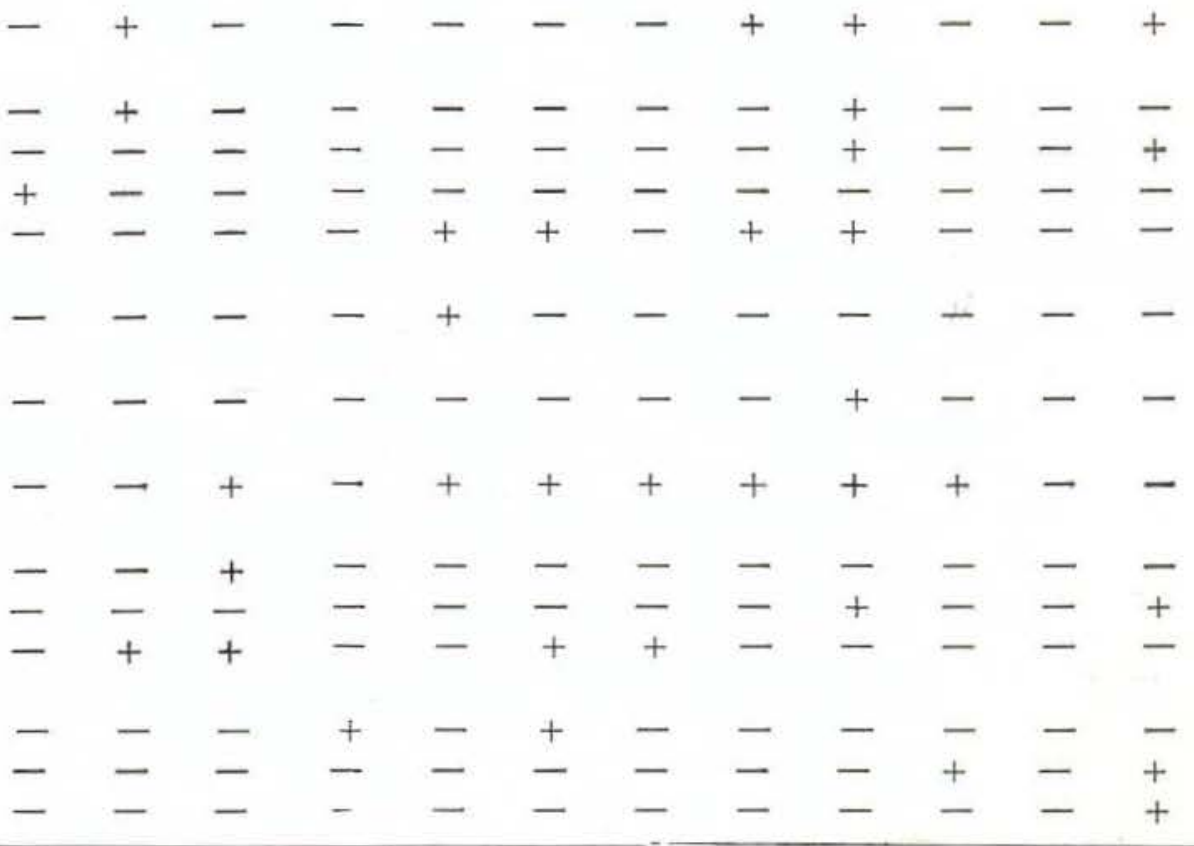

NOTA - As espécies assinaladas (*) foram também encontradas em Melipona seminigra.

TABELA II - Dados mensais para Melipona rufiventris

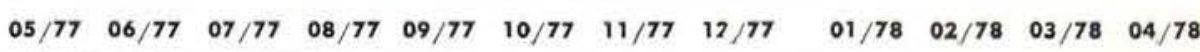

\section{AMARANTHACEAE}

- Alternanthera sp.

ANACARDIACEAE

* Anacardium occidentale

Spondias sp.

- Tapirira guianensis

ARALIACEAE

- Didymopanax morototoni BIXACEAE

* Bixa orellana BORAGINACEAE

Cordia sellowiana BURSERACEAE

* Protium heptaphyllum COMPOSITAE

Ageratum conyzoides

+ Melampodium camphoratum

Mikania roraimensis

Vernonia scabra

DILLENIACEAE

Doliocarpus spraguei EUPHORBIACEAE

Alchornea discolor

Croton lanjouwensis

* Mabea nitida

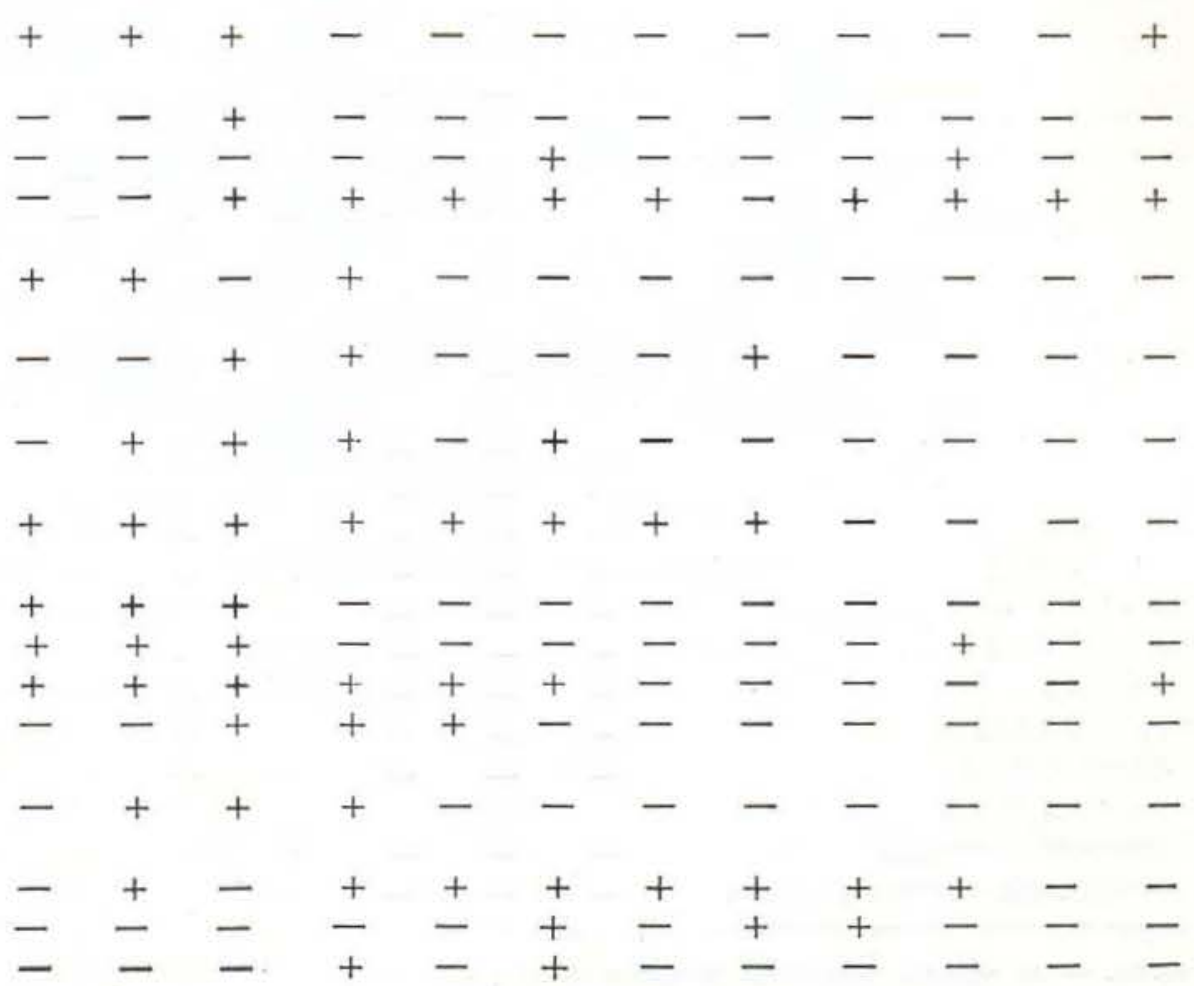

Plantas... 
FLACOURTIACEAE

" Casearia arborea GUTTIFERAE

Calophyllum sp.

* Vismia guianensis LABIATAE

" Hyptis brevipes LEG. CAESALP.

Cassia latifolia

- Cassia reticulata

" Cassia siamea LEG. MIMOS.

" Inga edulis

"Mimosa invisa

- Mimosa pudica

- Stryphnodendron guianensis LEG. PAP

- Aeschynomene sensitiva LORANTHACEAE

* Phthirusa micrantha MALPIGHIACEAE

* Byrsonima sp. MELASTOMATACEAE

* Bellucia grossularioides

* Bellucia imperialis

- Miconia sp. MYRTACEAE

- Eugenia sp.

* Syzygium jambolana PALMAE

" Maximiliana martiana RUBIACEAE

- Borreria capitata

* Borreria laevis

- Borreria latifolia

* Warszewiczia coccinea SAPINDACEAE

- Talisia sp. ULMACEAE

- Trema micrantha LABIATAE LAURACEAE

- leguminosae MYRTACEAE

PALMAE

- pRoteaceaE SAPINDACEAE Indeterminados:

- Diporado escabrado Tricolporado reticulado tipo 1 MORACEAE

Cecropia sp. COMPOSITAE "Ambrosia tipo"

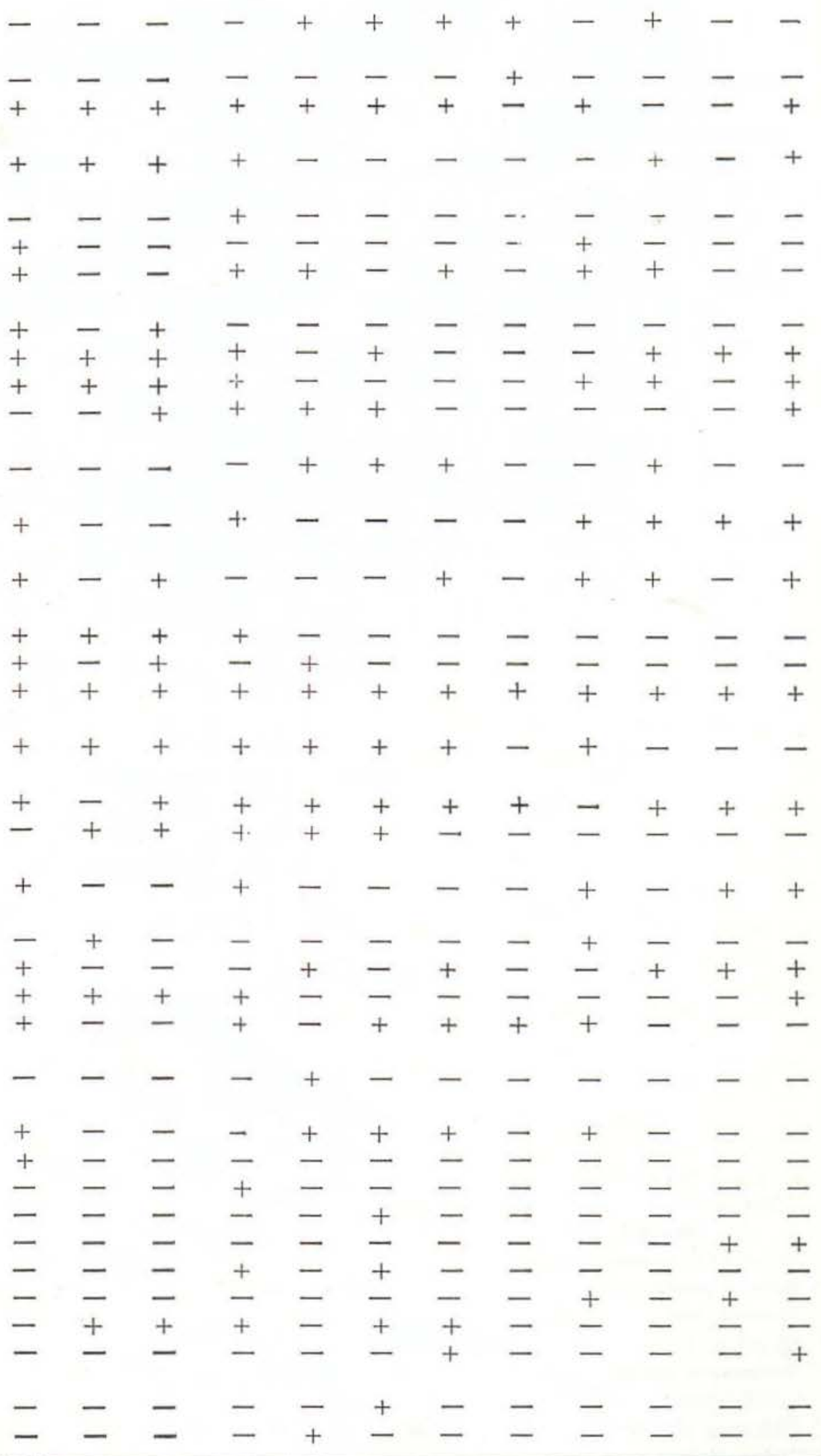

NOTA - As espécies assinaladas $\left({ }^{\circ}\right)$ foram também encontradas em Melipoma rufiventris. 
As plantas visitadas por Melipona seminigra foram as seguintes:

- 1 - Aeschynomene sensitiva Rudd (setembro, outubro, janeiro)

* 2 - Ageratum conyzoides L. (junho, julho)

- 3 - Alchornea discolor Poepp. \& Endl. (maio, junho, setembro a janeiro)

* 4 - Alternanthera sp. (junho, julho, setembro a novembro, fevereiro, abril)

* 5 - Anacardium occidentale DC. (setembro)

* 6 - Bellucia grossularioides Triana (maio a setembro)

* 7 - Bellucia imperialis Sald. \& Cogn. (maio a julho)

- 8 - Bixa orellana L. (outubro, novembro, janeiro, fevereiro, abril)

* 9 - Borreria capitata DC. (junho, janeiro)

*10 - Borreria laevis Griseb (janeiro, abril)

*11 - Borreria latifolia (Aubl.) K. Sch. (maio)

"12 - Byrsonima sp. (maio, outubro, novembro, janeiro, março)

*13 - Casearia arborea Urb. (novembro, dezembro)

"14 - Cassia reticulata Willd. (maio, junho, dezembro)

"15 - Cassia siamea Lam. (maio, setembro, janeiro a abril)

"16 - Cecropia sp. (maio, junho, julho, outubro a abril)

17 - Clusia aff. nemorosa G.F.W. Meyer (dezembro, janeiro)

"18 - Cordia sellowiana Cham. (junho, julho)

*19 - Croton lanjouwensis Jablonski (dezembro, janeiro)

*20 - Didymopanax morototoni Marchal. (junho)

"21 - Eugenia sp. (maio, junho, setembro a dezembro, janeiro, fevereiro, março, abrii)

22 - Hevea brasiliensis (Ule) Ducke Muell. Arg. (dezembro)

"23 - Hyptis brevipes Poit (maio, junho, agosto, setembro, abril)
"24 - Inga edulis Mart. (maio, junho, julho, agosto, abril)

*25 - Mabea nitida Spruce (outubro)

"26 - Maximiliana martiana Karst. (junho, dezembro, janeiro, abril)

"27 - Melampodium camphoratum Benth. \& Hook (abril)

*28 - Miconia sp. (maio, junho, julho, agosto, setembro, outubro, novembro. dezembro, janeiro, fevereiro, março, abril)

*29 - Mikania roraimensis Robison fjulho, abril)

"30 - Mimosa invisa Mart. (maio, junho, março, abril)

"31 - Mimosa pudica L. (maio, junho, janeiro, fevereiro, março, abril)

*32 - Phthirusa micrantha Eichl. (abril)

*33 - Protium heptaphyllum March. (maio a dezembro, fevereiro a abril)

34 - Rauwolfia grandiflora Mart. (novembro)

35 - Solanum sp. (janeiro)

36 - Stachytarpheta cayennensis Chod. (juIho)

"37 - Stryphnodendron guianensis Ducke. (maio, junho, setembro)

*38 - Syzygium jambolana DC. (junho)

*39 - Talisia sp. (setembro)

"40 - Tapirira guianensis Engler (maio, agosto, setembro, outubro)

*41 - Trema micrantha Blume (julho, setembro a dezembro, janeiro, fevereiro)

"42 - Vernonia scabra Moore (julho, agosto, setembro, outubro)

*43 - Vismia guianensis Choisy (maio, agosto, setembro, outubro, novembro, abril)

"44 - Warszewiczia coccinea Klotzsch (setembro, outubro, dezembro, janeiro)

" 45 - Leguminosae (janeiro, abril)

"46 - Proteaceae (junho, julho, outubro, novembro)

" 47 - Diporado escabrado (agosto, outubro)

48 - Tricolporado reticulado tipo 2 (fevereiro, abril)

49 - Tricolporado reticulado tipo 3 (abril)

NOTA: As 42 espécies assinaladas (") foram também encontradas em Melipona rufiventris. 
As plantas visitadas por Melipona rufiventris foram as seguintes:

* 1 - Aeschynomene sensitiva Rudd (setembro, outubro, novembro, fevereiro)

- 2 - Ageratum conyzoides L. (maio. junho, julho)

* 3 -- Aichornea discolor Poepp. \& Endl. (ju. nino, agosto a fevereiro)

* 4 - Alternanthera sp. (maio, junho, julho, abril)

* 5 - Anacardium occidentale DC. (julho)

* 6 - Bellucia grossularioides Triana (maio a agosto)

* 7 - Bellucia imperialis Sald. \& Cogn. (maio. julho, setembro)

" 8 - Bixa orellana L. (julho, agosto, dezembro)

* 9 - Borreria capitata DC. (junho, janeiro)

*10 - Borreria laevis Griseb. (maio, setembro, novembro, fevereiro a abril)

"11 - Borreria latifolia (Aubl.) K.Sch. (maio, junho, julho, agosto, abril)

"12 - Byrsonima sp. (maio, julho, novembro, janeiro, fevereiro, abril)

13 - Calophyllum sp. (dezembro)

*14 - Casearia arborea Urb. (setembro a dezembro, fevereiro]

15 - Cassia latifolia G.F.W. Meyer (agosto)

"16 - Cassia reticulata Willd. (maio, janeiro)

"17 - Cassia siamea Lam. (maio, agosto, setembro, novembro, janeiro, fevereiro)

"18 - Cecropia sp. (maio a novembro, janeiro)

"19 - Cordia sellowiana Cham. (junho, julho, agosto, outubro)

"20 - Croton lanjouwensis Jablonski (outubro, dezembro, janeiro)

"21 - Didymopanax morototoni Marchal. (maio, junho, agosto)

22 - Doliocarpus spraguei Cheesman. (junho, julho, agosto)

"23 - Eugenia sp. (maio, julho, agosto a dezembro, fevereiro a abril)

"24 - Hyptis brevipes Poit. (maio a agosto, fevereiro, abril)

"25 — Inga edulis Mart. (maio, julho)
"26 - Mabea nitida Spruce (agosto, outubro,

"27 - Maximiliana martiana Karst. (maio, agosto, janeiro, março, abril)

"28 - Melampodium camphoratum Benth. \& Hook (maio, junho, julho, fevereiro)

"29 - Miconia sp. (maio a dezembro, janeiro a abril)

"30 - Mikania roraimensis Robinson (maio a outubro, abril)

"31 - Mimosa invisa Mart. (maio a agosto, outubro, fevereiro a abril)

"32 - Mimosa pudica L. (maio a agosto, ja. neiro, fevereiro, abril)

"33 - Phthirusa micrantha Eichl. (maio, agos. to, janeiro a abril)

"34 - Protium heptaphyllum March. (maio a dezembro, janeiro a abril)

35 - Spondias sp. (outubro, fevereiro)

*36 - Stryphnodendron guianensis Ducke. (juIho a outubro, abril)

"37 - Syzygium jambolana DC. (junho a outubro]

*38 - Talisia sp. (setembro)

"39 - Tapirira guianensis Engler. (julho a novembro, janeiro a abril)

"40 - Trema micrantha Blume (maio, setembro, outubro, novembro, janeiro, fevereiro)

"41 - Vernonia scabra Moore (julho, agosto setembro)

"42 - Vismia guianensis Choisy (maio a novembro, janeiro, abril)

"43 - Warszewiczia coccinea Klotzsch (maio, agosto, outubro a janeiro)

44 - Compositae "Ambrosia tipo" (maio)

45 - Labiatae (agosto)

46 - Lauraceae (outubro)

*47 - Leguminosae (março, abril)

48 - Myrtaceae (agosto, outubro)

49 - Palmae (janeiro, março)

"50 - Proteaceae (junho, julho, agosto, outu. bro, novembro)

51 - Sapindaceae (novembro, abril)

"52 - Diporado escabrado (outubro)

53 - Tricolporado reticulado tipo 1 (setem. bro)

NOTA: As 42 espécies assinaladas (") foram também encontradas em Melipona seminigra. 

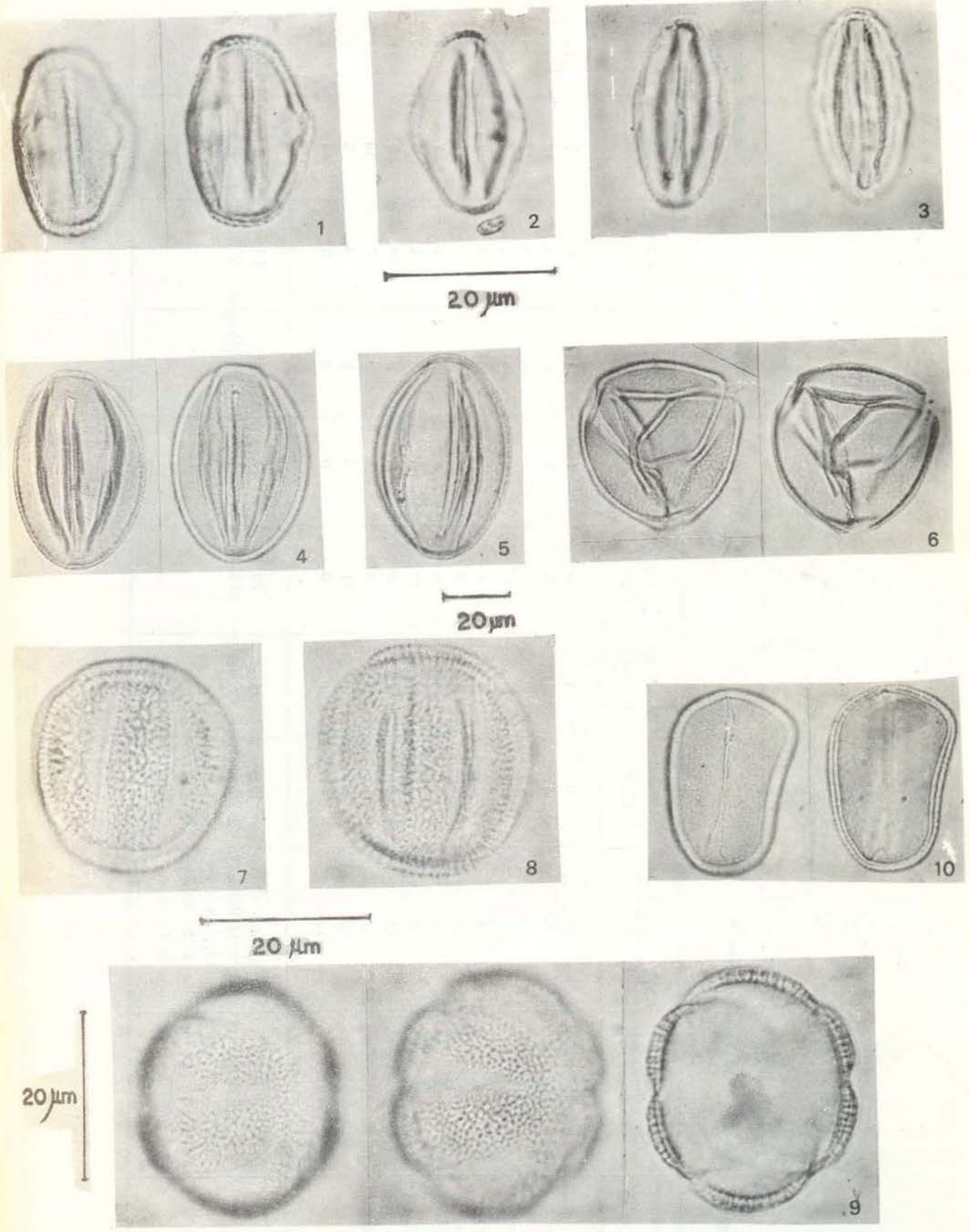

Est. I - Fig. 1-3 - Bellucia grossularioides (Melastomataceae); Fig. 4.6 - Cassia siamea (Leguminosae-Caesalp.); Fig. 7-9 - Hyptis brevipes (Labiatae); Fig. 10 - Maximiliana martiana (Palmae). 
TABELA III - Distribuição mensal dos tipos polínicos

\begin{tabular}{|c|c|c|c|c|c|c|c|c|c|c|}
\hline \multirow[t]{2}{*}{ M E S } & \multicolumn{2}{|c|}{$\begin{array}{l}\text { N. } 0 \text { mínimo de espécies } \\
\text { visitadas }\end{array}$} & \multicolumn{2}{|c|}{ Sem pólen } & \multicolumn{2}{|c|}{$\begin{array}{l}\text { Abelhas com } 1 \text { só } \\
\text { tipo de pólen }\end{array}$} & \multicolumn{2}{|c|}{$\begin{array}{l}\text { Abelhas com } 2 \\
\text { tipos de pólen }\end{array}$} & \multicolumn{2}{|c|}{$\begin{array}{l}\text { Abelhas com } 3 \\
\text { ou }+ \text { tip. pólen }\end{array}$} \\
\hline & M. seminigra & M. rufiventris & $\begin{array}{l}\text { M. semi- } \\
\text { nigra }\end{array}$ & $\begin{array}{l}\text { M. rufi- } \\
\text { ventris }\end{array}$ & $\begin{array}{l}\text { M. semi- } \\
\text { nigra }\end{array}$ & $\begin{array}{l}\text { M. rufi- } \\
\text { ventris }\end{array}$ & $\begin{array}{l}\text { M. semi- } \\
\text { nigra }\end{array}$ & $\begin{array}{l}\text { M. rufi- } \\
\text { ventris }\end{array}$ & $\begin{array}{l}\text { M. semi- } \\
\text { nigra }\end{array}$ & $\begin{array}{l}\text { M. rufi- } \\
\text { ventris }\end{array}$ \\
\hline MAIO & 18 & 26 & 2 & 1 & 6 & 1 & 11 & 3 & 7 & 21 \\
\hline JUNHO & 21 & 20 & 1 & 1 & 5 & 1 & 4 & 3 & 15 & 20 \\
\hline JULHO & 14 & 26 & 1 & 0 & 4 & 1 & 8 & 1 & 13 & 24 \\
\hline AGOSTO & 9 & 28 & 0 & 0 & 9 & 0 & 13 & 1 & 5 & 26 \\
\hline SETEMBRO & 17 & 19 & 1 & 0 & 4 & 4 & 4 & 1 & 16 & 20 \\
\hline OUTUBRO & 17 & 23 & 2 & 1 & 5 & 3 & 8 & 1 & 11 & 21 \\
\hline NOVEMBRO & 13 & 16 & 2 & 0 & 6 & 5 & 9 & 5 & 7 & 14 \\
\hline DEZEMBRO & 13 & 9 & 5 & 7 & 12 & 6 & 4 & 8 & 5 & 5 \\
\hline JANEIRO & 18 & 18 & 0 & 1 & 8 & 4 & 9 & 10 & 9 & 11 \\
\hline FEVEREIRO & 10 & 16 & 2 & 0 & 4 & 4 & 6 & 4 & 10 & 14 \\
\hline MARÇO & 8 & 9 & 0 & 0 & 11 & 4 & 8 & 9 & 6 & 12 \\
\hline ABRIL & 20 & 18 & 0 & 0 & 6 & 0 & 4 & 8 & 14 & 16 \\
\hline TOTAL & $\begin{array}{l}\text { Um mínimo de } 49 \\
\text { tipos polínicos }(7 \\
\text { gêneros, } 37 \text { espé- } \\
\text { cies e } 3 \text { não de- } \\
\text { terminaćos) }\end{array}$ & $\begin{array}{l}\text { Um mínimo de } 53 \\
\text { tipos polínicos }(8 \\
\text { gêneros, } 35 \text { espé- } \\
\text { cies e } 2 \text { indeter. } \\
\text { minados) }\end{array}$ & $\begin{array}{l}16 \\
5,3 \%\end{array}$ & $\begin{array}{l}11 \\
3,6 \%\end{array}$ & $\begin{array}{l}80 \\
28 \%\end{array}$ & $\begin{array}{l}33 \\
11,3 \%\end{array}$ & $\begin{array}{l}88 \\
30,8 \%\end{array}$ & $\begin{array}{l}54 \\
18,6 \%\end{array}$ & $\begin{array}{l}118 \\
41,2 \%\end{array}$ & $\begin{array}{l}204 \\
70,1 \%\end{array}$ \\
\hline
\end{tabular}


TABELA IV - Dados mensais de abelhas com e sem coleta de néctar e precipitação pluviométrica mensal respectiva.

\begin{tabular}{|c|c|c|c|c|c|c|c|c|c|c|c|c|c|c|c|c|c|c|c|c|c|c|c|c|c|}
\hline \multirow{2}{*}{1977 / 1978} & \multicolumn{2}{|c|}{ MAIO } & \multicolumn{2}{|c|}{ JUN. } & \multicolumn{2}{|c|}{ JUL. } & \multicolumn{2}{|c|}{ AGO. } & \multicolumn{2}{|c|}{ SET. } & \multicolumn{2}{|c|}{ out. } & \multicolumn{2}{|c|}{ NOV. } & \multicolumn{2}{|c|}{ DEZ. } & \multicolumn{2}{|c|}{ JAN. } & \multicolumn{2}{|c|}{ FEV. } & \multicolumn{2}{|c|}{ MAR. } & \multicolumn{2}{|c|}{ ABR. } & \multirow{2}{*}{$\begin{array}{l}\text { TOTAL } \\
\text { GERAL } \\
\text { (Dados } \\
\text { anuais) }\end{array}$} \\
\hline & M.S & M.R & M.S & M.R & M.S & M.R & M.S & M.R & M.S & M.R & M.S & M.R & M.S & M.R & M.S & M.R & M.S & M.R & M.S & M.R & M.S & M.R & M.S & M.R & \\
\hline $\begin{array}{l}\mathrm{N} . \text { de amostras } \\
\text { coletadas }\end{array}$ & 26 & 26 & 25 & 25 & 26 & 26 & 27 & 27 & 25 & 25 & 26 & 26 & 24 & 24 & 26 & 26 & 26 & 26 & 22 & 22 & 25 & 25 & 24 & 24 & 604 \\
\hline $\begin{array}{l}\text { Total de amostras } \\
\text { com pólen }\end{array}$ & 24 & 25 & 24 & 24 & 25 & 26 & 27 & 27 & 24 & 25 & 24 & 25 & 22 & 24 & 21 & 19 & 26 & 25 & 20 & 22 & 25 & 25 & 24 & 24 & 577 \\
\hline $\begin{array}{l}\% \text { de amostras } \\
\text { com pólen }\end{array}$ & 92,3 & 96,2 & 96 & 96 & 96,2 & 100 & 100 & 100 & 96 & 100 & 92,3 & 96,2 & 91.7 & 100 & 80,8 & 73.1 & 100 & 96,2 & 90,9 & 100 & 100 & 100 & 100 & 100 & 95,5 \\
\hline $\begin{array}{l}\text { Total de amostras } \\
\text { sem pólen }\end{array}$ & 2 & 1 & 1 & 1 & 1 & 0 & 0 & 0 & 1 & 0 & 2 & 1 & 2 & 0 & 5 & 7 & 0 & 1 & 2 & 0 & 0 & 0 & 0 & 0 & 27 \\
\hline $\begin{array}{l}\% \text { de amostras } \\
\text { sem pólen }\end{array}$ & 7,7 & 3.8 & 4 & 4 & 3,8 & 0 & 0 & 0 & 4 & 0 & 7,7 & 3,8 & 8,3 & a & 19,2 & 26,9 & 0 & 3,8 & 9,1 & 0 & 0 & $B$ & 0 & 0 & 4,5 \\
\hline Precipitaçăo $(\mathrm{mm})$ & \multicolumn{2}{|c|}{267.8} & \multicolumn{2}{|c|}{98.4} & \multicolumn{2}{|c|}{79.9} & \multicolumn{2}{|c|}{23.0} & \multicolumn{2}{|c|}{165.8} & \multicolumn{2}{|c|}{275.8} & \multicolumn{2}{|c|}{149.6} & \multicolumn{2}{|c|}{262.4} & \multicolumn{2}{|c|}{277.9} & \multicolumn{2}{|c|}{218.5} & \multicolumn{2}{|c|}{366.6} & \multicolumn{2}{|c|}{334.1} & 2.519 .8 \\
\hline
\end{tabular}

M.S = Melipona seminigra

$M \cdot R=$ Melipona rufiventris 

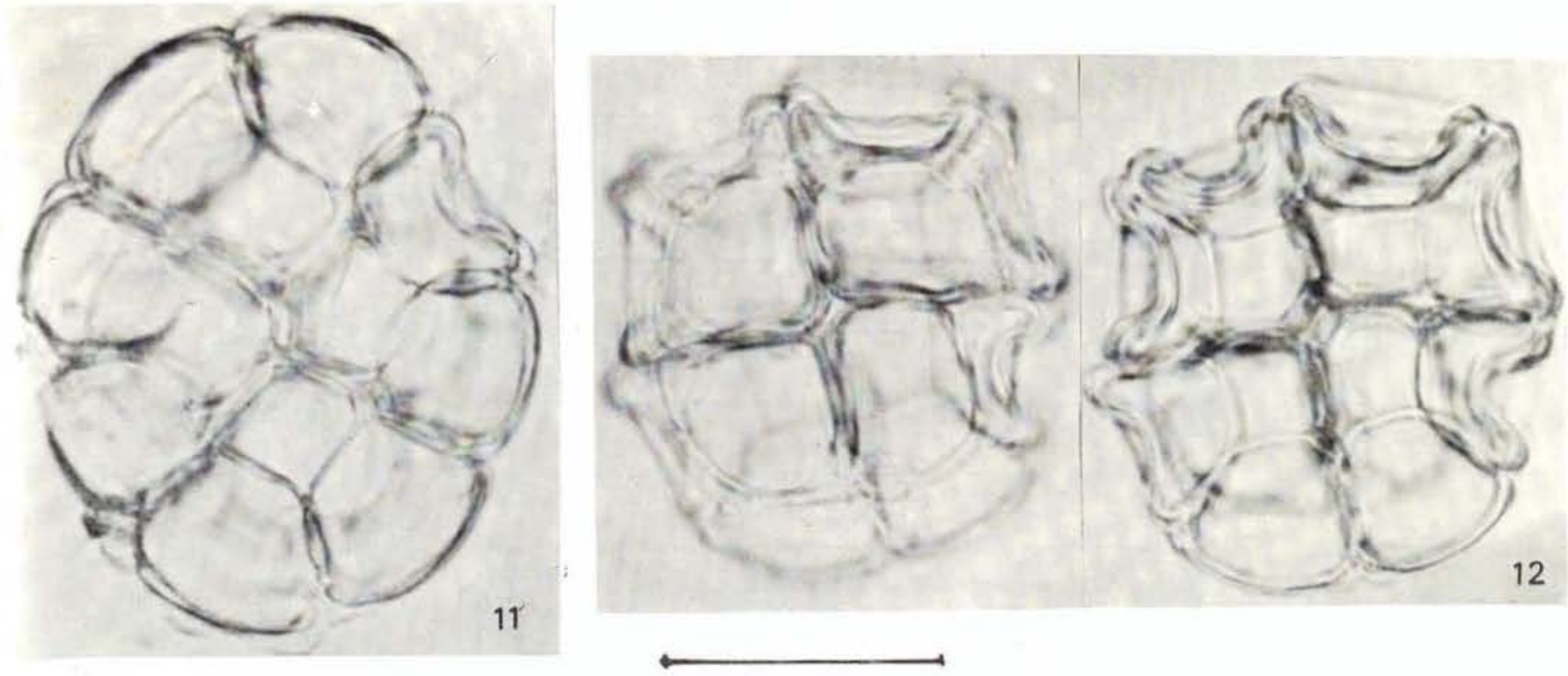

$20 \mu \mathrm{m}$
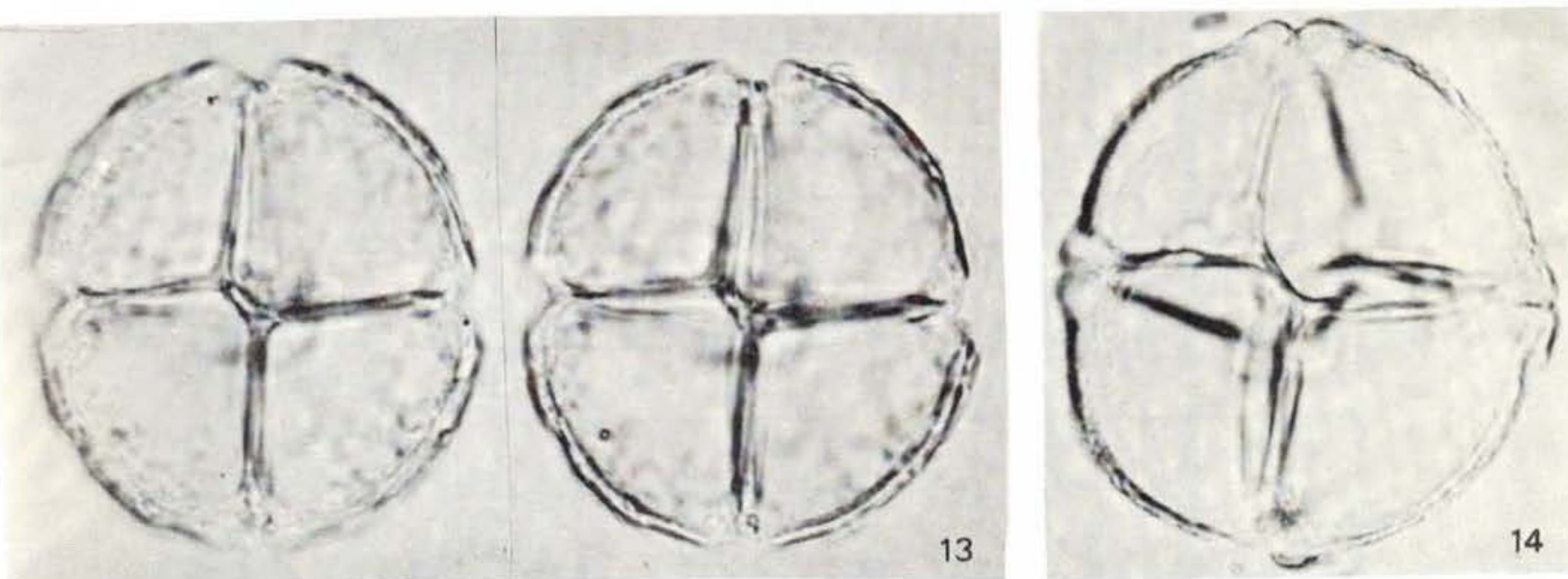

Est. II - Fig. 11-12 - Stryphnodendron guianensis (Leguminosae-Mimos.); Fig. 13-14 - Mimosa invisa (Leguminosae-Mimos.).

\section{Discussão}

A tabela III indica que $28 \%$ das operárias de Melipona seminigra e $11,3 \%$ de Melipona rufiventris que foram observadas, visitaram apenas uma espécie de flor, enquanto que $30,8 \%$ de $M$. seminigra e $18,6 \%$ de $M$. rufiventris visitaram duas espécies e $41,2 \%$ de $M$. seminigra e $70,1 \%$ de $M$. rufiventris visitaram três ou mais espécies de flores; $5,3 \%$ de $M$. seminigra e $3,6 \%$ de $M$. rufiventris não colheram pólen.
Como no trabalho anterior, a tabela IV é uma tentativa de correlacionar a freqüência de abelhas com pólen ou sem pólen e a precipitação pluviométrica (obtida de Ribeiro, 1977 e 1978) sem resultado. O total de abelhas com pólen foi de $95,5 \%$ e as sem pólen foi de $4,5 \%$. Os meses de maior precipitação foram outubro, janeiro, março e abril, nos quais tivemos tanto as maiores $(96,2 ; 100 \%)$ quanto as menores $(92,3 \%)$ freqüências de abelhas com pólen. O mesmo se observa nos meses de mais seca (junho, julho, agosto, novembro). Dessa 
forma, conclui-se que a coleta de pólen está ligada às épocas de floração das diferentes espéries e não apresenta correlação com a precipitação pluviométrica.

Além de indicar para os meliponicultores da área 60 espécies de plantas nectaríferas estudadas, constata-se que:

a) Das 49 espécies que são visitadas por Melipona seminigra 7 não são comuns à $M e l i$ pona rufiventris e das 53 visitadas por Melipona rufiventris 11 não são visitadas por Melipona seminigra. Como as colmeias estavam situadas no INPA (Manaus) e, portanto as operárias exploravam uma mesma comunidade de plantas, podemos dizer que as 7 plantas, não visitadas por Melipona rufiventris e as 11 não visitadas por Melipona seminigra, são as que tornam o nicho ecológico de uma diferente da outra, satisfazendo assim ao princípio de Gause (1934) que reza que duas espécies não podem ocupar o mesmo nicho ecológico; b) Cinco plantas da nossa lista anterior de 33 espécies não foram visitadas para néctar, a saber: Amanoa sp., Cordia, lanata, Eschweilera fracta, Mauritia flexuosa, Symmeria sp., isto é, são, possivelmente, plantas apenas poliníferas.

Cerca de 3 espécies, não identificadas, devem ser apenas nectaríferas, pois não continham pólen. Na Amazônia, há um grande número de plantas que não são hermafroditas (Kerr, 1978). Assim, é possível que se tenham desenvolvido nas plantas da Amazônia estratégias a fim de as plantas masculinas de uma espécie terem flores atrativas (para pólen e néctar) num certo período, a seguir, cessar de ser atrativa, de modo a obrigar as abelhas a irem procurar as flores femininas (para néctar). Com isso, os erros devem existir em maior número quando as abelhas procuram a parceira feminina, o que explicaria encontrarmos $61 \%$ das operárias coletando uma só espécie de pólen contra $28 \%$ visitando uma só espécie para néctar em $M$. seminigra (Absy \& Kerr, 1977).

\section{Agradecimentos}

Agradecemos o Dr. William A. Rodrigues e a Dra. Marlene F. da Silva pela identificação dos espécimens vegetais, o $\mathrm{Sr}$. Luis Fernandes Coelho pela ajuda na coleta do material botânico e a Sra. Barbara Bailey Gibbs pela reprodução das fotografias.

\section{SUMMARY}

During a period of one year samples of bee nectar from 302 Melipona seminigra merrillae Cock. and 302 Melipona rufiventris paraensis Ducke from Manaus (22-27) per month) were examined. Sixteen $(5,3 \%)$ samples from $M$. seminigra and $11(3,6 \%)$ samples from $M$. rufiventris did not contain any pollen. Of the $286(94,7 \%)$ remaining samples of $M$. seminigra, 80 $(20 \%)$ had one type of pollen, $88(30,8 \%)$ had two, and $118(41,2 \%)$ had 3 or more different types. Of the 291 $(96,4 \%)$ remaining samples from $M$. rufiventris, 33 $(11,3 \%)$ had pollen of a single species of plant, 54 $(18,6 \%)$ had two, and $204(70,1 \%)$ of three or more different types. A total of 60 different types of pollen from 29 families, 42 genera, and 39 species of plants were recognized.

It is suggested that the greater frequency of visits to more than one species in nectariferous flowers $(72 \%)$ than to polliniferous ones $(39 \%)$ is due to strategies developed in order to make the bees visit male flowers for pollen and later the females, for nectar.

\section{BIBLIOGRAFIA}

ABSY, M.L

1979 - A palynological study of Holocene sediments in the Amazon basin. Ph. D. Tese. Universidade de Amsterdam.

ABSY, M.L. \& KERR, W.E.

1977 - Algumas plantas visitadas para obtenção de pólen por operárias de Melipona seminigra merrillae em Manaus. Acta Amazonica, $7(3): 309-315$.

CARREIRA, L.M.M.

1976 - Morfologia polínica de plantas lenhosas da Campina. Tese. Acta Amazonica, 6(3) : 247-269.

ERDTMAN, G.

1960 - The acetolysis method: - a revised description. Sv. Bot. Tidskr., Lund, 54(4) : 561-564.

Gause, G.F.

1934 - The struggle for Existence. Dover Publications, Inc. New York, 163 p. ilust. ediçăo inalterada de 1971 .

KERR, W.E.

1978 - Papel das abelhas sociais na Amazônia. In: Simpósio Internacional "Apicultura em clima quente", p. 119-129. Bucareste, Editora Apimonolia. 
KerR, W.E.; Sakagami, S.F.; Zucchi, R.; PortugalAraúJo, V. dE \& Camargo, J.M.F.

1967 - Observações sobre a arquitetura dos ninhos e comportamento de algumas espécies de abelhas sem ferrão das vizinhanças de Manaus, Amazonas (Hymenoptera, Apoide2). Atas Simp. Biota Amazônica, 5 (Zool.) : 255-309.
RIBEIRO, M.N.G.

$\begin{aligned} 1977 \text { - } & \text { Dados meteorológicos coletados na Esta- } \\ & \text { ção Meteorológica na Reserva Ducke - Km } \\ & 26 \text { da Rodovia Torquato Tapajós. Bol. Me- } \\ & \text { teorológico, INPA, Manaus. jan./dez. } \\ 1978 \text { - } & \text { Boletim Meteorológico, INPA, Manaus. } \\ & \text { jan./jun. }\end{aligned}$

(Aceito para publicação em 29/02/80) 